International Journal of Applied Mathematics

Volume 33 No. $3 \quad 2020,439-450$

ISSN: 1311-1728 (printed version); ISSN: 1314-8060 (on-line version)

doi: http://dx.doi.org/10.12732/ijam.v33i3.6

\title{
ON QUARTER-SWEEP FINITE DIFFERENCE SCHEME FOR ONE-DIMENSIONAL POROUS MEDIUM EQUATIONS
}

\author{
Jackel Chew Vui Lung ${ }^{1 \S}$, Jumat Sulaiman ${ }^{2}$ \\ ${ }^{1}$ Faculty of Computing and Informatics \\ Universiti Malaysia Sabah Labuan International Campus \\ 87000, Labuan F.T., MALAYSIA \\ ${ }^{2}$ Faculty of Science and Natural Resource \\ Universiti Malaysia Sabah \\ 88400, Kota Kinabalu, Sabah, MALAYSIA
}

\begin{abstract}
In this article, we introduce an implicit finite difference approximation for one-dimensional porous medium equations using Quarter-Sweep approach. We approximate the solutions of the nonlinear porous medium equations by the application of the Newton method and use the Gauss-Seidel iteration. This yields a numerical method that reduces the computational complexity when the spatial grid spaces are reduced. The numerical result shows that the proposed method has a smaller number of iterations, a shorter computation time and a good accuracy compared to Newton-Gauss-Seidel and Half-Sweep Newton-Gauss-Seidel methods.
\end{abstract}

AMS Subject Classification: 65M06, 65M22

Key Words: porous medium equations; finite difference method; QuarterSweep, Newton, Gauss-Seidel methods

\section{Introduction}

In the present article, we consider an implicit finite difference approximation using Quarter-Sweep approach for the one-dimensional porous medium equations (1D PME) of the form,

Received: February 12, 2020

(c) 2020 Academic Publications

${ }^{\S}$ Correspondence author 


$$
\frac{\partial u}{\partial t}=C \frac{\partial}{\partial x}\left(u^{m} \frac{\partial u}{\partial x}\right),
$$

that is subject to the initial and boundary conditions as follows:

$$
\begin{gathered}
u(x, 0)=f_{0}(x), \quad 0 \leq x \leq 1, \\
u(0, t)=g_{0}(t), u(1, t)=g_{1}(t), \quad 0 \leq t \leq 1,
\end{gathered}
$$

with rational numbers, $C$ and $m$.

The partial differential equation (1) arises, for example, in flow of incompressible fluids, gas flow in porous media, nonlinear process of heat transfer and image processing. Equation (1) itself represents a nonlinear diffusion process. It has many interesting features and one of them is the finite propagation induced by the degeneracy. When the initial condition $u(x, 0)$ has compact support, the solution will have a compact support for any time $t>t_{0}$. This creates a free boundary between the regions where the solution $u$ is nonzero and where $u$ vanishes (propagates at a finite speed for any time). Moreover, one of the obvious differences between equation (1) and its closest variant, the heat equation, is the heat equation can smooth out its initial solutions while the solution of equation (1) can become non-smooth even in cases where it has a smooth initial solution. For more details, see Vazquez [7].

Since the introduction of Quarter-Sweep approach by Othman and Abdullah [10], it has been studied by few researchers which can be referred in Eng et al. [5], Sunarto and Sulaiman [1], Suardi et al. [11], Aruchunan et al. [4], and Nusi and Othman [12]. In contrast to these works, in the present article, we attempt to apply the Quarter-Sweep into the formulation of finite difference approximation for 1D PME. To approximate the solutions of 1D PME, we use the combination of Newton method and Gauss-Seidel iteration which eventually become a new iterative method that can be abbreviated as QSNGS method.

This article is outlined as follows. In Section 2, we present the formulation of the implicit finite difference approximation equation using Quarter-Sweep for equation (1). Then, we show the derivation of the Newton-Gauss-Seidel method for equation (1) from the formulated approximation equation. Section 3 illustrates the numerical results from the implementation of QSNGS on some examples of 1D PME problems. As a comparison, we selected the methods: the Newton-Gauss-Seidel (NGS) and the Half-Sweep version of Newton-GaussSeidel (HSNGS) from Chew [9]. Section 4 concludes the finding of the article. 


\section{Methodology}

In this section, we show the Quarter-Sweep finite difference approximation of equation (1) and the Newton-Gauss-Seidel method for the approximate solution. To begin, we define the difference operators:

$$
\begin{gathered}
\frac{\partial u}{\partial t}=\frac{U_{p, n+1}-U_{p, n}}{k} \\
\frac{\partial^{2} u}{\partial x^{2}}=\frac{U_{p+4, n+1}-2 U_{p, n+1}+U_{p-4, n+1}}{(4 h)^{2}} \\
\frac{\partial u}{\partial x}=\frac{U_{p+4, n+1}-U_{p-4, n+1}}{8 h} .
\end{gathered}
$$

We use the following spatial and temporal steps as follows: Let $M$ and $N$ be natural numbers and $h=\frac{1}{M}, k=\frac{1}{N}, x_{p}=p h, p=0, . ., M, t_{n}=n k$ and $t_{n+1}=$ $(n+1) k$. The numerical solution at time level $n$ and $n+1$ are denoted as $U_{p, n}=$ $U\left(x_{p}, t_{n}\right)$ and $U_{p, n+1}=U\left(x_{p}, t_{n+1}\right)$ respectively. The corresponding solution vectors can be denoted as $U_{n}=\left(U_{0, n}, . ., U_{M, n}\right)$ and $U_{n+1}=\left(U_{0, n+1}, . ., U_{M, n+1}\right)$. To perform the discretization of equation (1) using equation (4-6), we find the derivatives for each term in equation (1) and write it into

$$
\frac{\partial u}{\partial t}=C u^{m} \frac{\partial^{2} u}{\partial x^{2}}+C m u^{m-1}\left(\frac{\partial u}{\partial x}\right)^{2},
$$

then we discretize equation (7) in order to form the Quarter-Sweep finite difference approximation of equation (1) and yields

$$
f_{p, n+1}=U_{p, n+1}-\alpha A_{1}+2 \alpha A_{2}-\alpha A_{3}-\beta A_{4}+2 \beta A_{5}-\beta A_{6}-U_{p, n},
$$

where

$$
\begin{gathered}
\alpha=\frac{C k}{16 h^{2}}, \beta=\frac{C k}{64 h^{2}}, p=4,8, . ., M-8, M-4, \\
A_{1}=U_{p, n+1}^{m} U_{p+4, n+1}, A_{2}=U_{p, n+1}^{m+1}, A_{3}=U_{p, n+1}^{m} U_{p-4, n+1}, \\
A_{4}=m U_{p, n+1}^{m-1} U_{p+4, n+1}^{2}, A_{5}=m U_{p, n+1}^{m-1} U_{p+4, n+1} U_{p-4, n+1}, \\
A_{6}=m U_{p, n+1}^{m-1} U_{p-4, n+1}^{2} \text { and } n=0, . ., N-1 .
\end{gathered}
$$

We investigate the implementation of equation (8) on a solution domain of equation (1) that contains $M$ grid points. When equation (8) is applied to each 
of the grid points in the solution domain, it will result in a large-sized system of nonlinear equations that has a form of

$$
\left[\begin{array}{c}
f_{4, n+1}\left(U_{n+1}\right) \\
f_{8, n+1}\left(U_{n+1}\right) \\
\vdots \\
f_{M-8, n+1}\left(U_{n+1}\right) \\
f_{M-4, n+1}\left(U_{n+1}\right)
\end{array}\right]=0
$$

To solve the system of nonlinear equations (9), we derive the Newton-GaussSeidel method which has been discussed in Ortega and Rheinboldt [8] and More [6]. First, to derive the Newton method for a system of nonlinear equations, we find the Jacobian matrix with dimension $(M-4) \times(M-4)$ as follows,

$$
\left[\begin{array}{ccc}
\frac{\partial f_{4, n+1}}{\partial U_{4, n+1}} & \cdots & \frac{\partial f_{4, n+1}}{\partial U_{M-4, n+1}} \\
\vdots & & \vdots \\
\frac{\partial f_{M-4, n+1}}{\partial U_{4, n+1}} & \cdots & \frac{\partial f_{M-4, n+1}}{\partial U_{M-4, n+1}}
\end{array}\right] .
$$

By denoting $W_{(n+1)}=U_{(n+1)}^{(\ell+1)}-U_{(n+1)}^{(\ell)}$ and $\ell$ as the number of the currently executing iteration, we obtain the corresponding system of linear equations

$$
J_{n+1} W_{n+1}=-F\left(U_{n+1}\right),
$$

where $W_{n+1}$ and $F\left(U_{n+1}\right)$ are both column matrices with dimension $(M-4) \times 1$. Second, using equation (10), we separate the Jacobian matrix into three parts as follows

$$
J_{n+1}=D_{n+1}+L_{n+1}+V_{n+1},
$$

where $D_{n+1}$ is the diagonal part, $L_{n+1}$ is the lower triangular part and $V_{n+1}$ is the upper triangular part. The entries for the diagonal part are all nonzero. We substitute equation (12) into equation (11) and rearrange it to get the iteration form of QSNGS:

$$
W_{n+1}^{(\ell+1)}=\left(D_{n+1}+L_{n+1}\right)^{-1}\left(-V_{n+1} W_{n+1}^{(\ell)}-F\left(U_{n+1}\right)\right),
$$

The implementation of the QSNGS can be outlined as follows.

\section{Algorithm 1:}

i. Set the initial vector $U_{n+1}=1.0000$ and the tolerance error $\varepsilon=10^{-10}$,

ii. Compute $J_{n+1}, W_{n+1}$ and $F\left(U_{n+1}\right)$, 
iii. Iterate Eq. (11),

iv. $\left|W_{n+1}^{(\ell+1)}-W_{n+1}^{(\ell)}\right| \leq \varepsilon$,

v. Compute $U_{n+1}^{(\ell+1)}=U_{n+1}^{(\ell)}+W_{n+1}^{(\ell+1)}$,

vi. Check whether $\left|F\left(U_{n+1}^{(\ell+1)}\right)-F\left(U_{n+1}^{(\ell)}\right)\right| \leq \varepsilon$,

vii. Display the outputs such as the number of iterations, the computation time and the maximum absolute errors.

\section{Numerical experiments}

To study the performance of QSNGS method, we conduct a comparative analysis between the QSNGS and the other two tested methods, NGS and HSNGS. The performance analysis is studied by using three selected 1D PME problems and the criteria to be observed are the number of iterations $\left(\ell_{\max }\right)$, the computation time $(s)$ and the maximum absolute error $\left(\varepsilon_{\max }\right)$. The number of grid points $\mathrm{M}$ to be considered is 256, 512, 1024, 2048, and 4096. Below is the following 1D PME problems and the exact solutions to be tested.

Example 1: See in Polyanin and Zaitsev [2]

$$
\frac{\partial u}{\partial t}=\frac{\partial}{\partial x}\left(u \frac{\partial u}{\partial x}\right), \quad u(x, t)=x+t .
$$

Example 2: See in Wazwaz [3]

$$
\frac{\partial u}{\partial t}=\frac{\partial}{\partial x}\left(u^{2} \frac{\partial u}{\partial x}\right), \quad u(x, t)=\frac{x+1}{2 \sqrt{4-t}} .
$$

Example 3: See in Wazwaz [3]

$$
\frac{\partial u}{\partial t}=\frac{1}{2} \frac{\partial}{\partial x}\left(\frac{1}{u^{2}} \frac{\partial u}{\partial x}\right), \frac{1}{\sqrt{0.7 x-0.1225 t+1.35}} .
$$

The numerical output from the implementation of Algorithm 1 is collected and tabulated in Tables 1, 2 and 3 together with the numerical results from NGS and HSNGS from Chew [9]. The overall finding from the numerical experiment shows that QSNGS has successfully reduced the number of iterations and the computation time in computing the numerical solution of 1D PME when it is 
compared to NGS and HSNGS. The accuracy of the QSNGS is also as good as both NGS and HSNGS.

It can be said that by applying the Quarter-Sweep scheme in the formulation of the finite difference approximation to 1D PME, the computational complexity can be lowered in obtaining the solution of the nonlinear differential equation like PME. The reason is the implementation by NGS method computes all grid points in the solution domain. This causes the computational burden to be higher especially when the distance between grid points become narrower. As discussed in Chew [9], the combination of Half-Sweep with the finite difference scheme has shown its capability in reducing the computational complexity of the NGS by taking half of the total grid points. This paper, however, showed that the computational complexity can be reduced even further with the use of Quarter-Sweep that calculate only a quarter of all grid points in the solution domain, see Figure 1.

This finding is supported by the number of arithmetic operations per iteration as shown in Table 4. For instance, per iteration on a single point, QSNGS uses 2 operations for PLUS/MINUS and 3 operations for MULTIPLY/DIVIDE. Since the Quarter-Sweep scheme consider one-fourth of the grid points, thus amount of PLUS /MINUS and MULTIPLY/DIVIDE becomes $2\left(\frac{M}{4}-1\right)$ and $\left.3\left(\frac{M}{4}-1\right)\right)$ respectively.

\section{Conclusion}

Applied to the nonlinear one-dimensional porous medium equation, the QuarterSweep finite difference scheme and Newton-Gauss-Seidel has shown the capability in obtaining the numerical solutions with better efficiency. We have observed that the numerical solution obtained by the proposed method has a smaller number of iterations, a shorter computation time and a comparable accuracy when it is compared to Newton-Gauss-Seidel and Half-Sweep Newton-GaussSeidel. 


\section{Appendices}

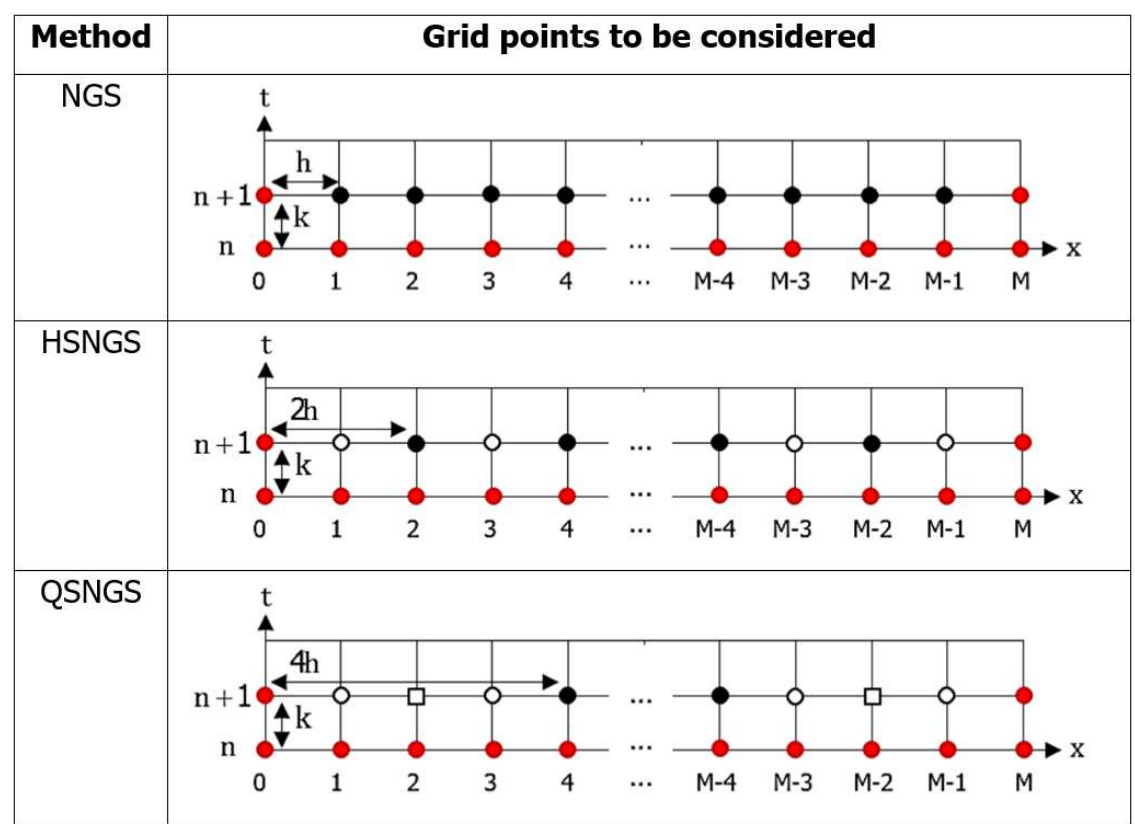

Figure 1: Comparison in terms of implementation by the three methods 
Table 1. Numerical result of Example 1

\begin{tabular}{rlrrc}
\hline$M$ & Method & \multicolumn{1}{c}{$\ell_{\max }$} & \multicolumn{1}{c}{$s$} & $\varepsilon_{\max }$ \\
\hline 256 & NGS & 48395 & 19.31 & $5.33 \mathrm{E}-07$ \\
& HSNGS & 13678 & 6.32 & $1.22 \mathrm{E}-07$ \\
& QSNGS & 3835 & 1.91 & $2.75 \mathrm{E}-08$ \\
\hline 512 & NGS & 169693 & 133.84 & $2.10 \mathrm{E}-06$ \\
& HSNGS & 48395 & 31.05 & $5.33 \mathrm{E}-07$ \\
& QSNGS & 13678 & 7.78 & $1.22 \mathrm{E}-07$ \\
\hline 1024 & NGS & 587031 & 919.22 & $7.62 \mathrm{E}-06$ \\
& HSNGS & 169693 & 244.23 & $2.10 \mathrm{E}-06$ \\
& QSNGS & 48395 & 44.79 & $5.33 \mathrm{E}-07$ \\
\hline 2048 & NGS & 1993096 & 6208.25 & $2.67 \mathrm{E}-05$ \\
& HSNGS & 587031 & 992.64 & $7.62 \mathrm{E}-06$ \\
& QSNGS & 169693 & 270.08 & $2.10 \mathrm{E}-06$ \\
\hline 4096 & NGS & 6612931 & 40998.73 & $9.66 \mathrm{E}-05$ \\
& HSNGS & 1993096 & 6697.98 & $2.67 \mathrm{E}-05$ \\
& QSNGS & 587031 & 2068.44 & $7.62 \mathrm{E}-06$ \\
\hline
\end{tabular}


Table 2. Numerical result of Example 2

\begin{tabular}{clrrc}
\hline$M$ & Method & \multicolumn{1}{c}{$\ell_{\max }$} & \multicolumn{1}{c}{$s$} & $\varepsilon_{\max }$ \\
\hline 256 & NGS & 17308 & 10.88 & $8.39 \mathrm{E}-05$ \\
& HSNGS & 4824 & 3.23 & $8.39 \mathrm{E}-05$ \\
& QSNGS & 1345 & 1.43 & $8.39 \mathrm{E}-05$ \\
\hline 512 & NGS & 61658 & 76.91 & $8.40 \mathrm{E}-05$ \\
& HSNGS & 17308 & 22.16 & $8.39 \mathrm{E}-05$ \\
& QSNGS & 4824 & 4.86 & $8.39 \mathrm{E}-05$ \\
\hline 1024 & NGS & 218147 & 557.97 & $8.43 \mathrm{E}-05$ \\
& HSNGS & 61658 & 173.79 & $8.40 \mathrm{E}-05$ \\
& QSNGS & 17308 & 27.22 & $8.39 \mathrm{E}-05$ \\
\hline 2048 & NGS & 763998 & 3839.51 & $8.55 \mathrm{E}-05$ \\
& HSNGS & 218147 & 950.34 & $8.43 \mathrm{E}-05$ \\
& QSNGS & 61658 & 192.19 & $8.40 \mathrm{E}-05$ \\
\hline 4096 & NGS & 2630914 & 26497.28 & $8.99 \mathrm{E}-05$ \\
& HSNGS & 763998 & 4136.48 & $8.55 \mathrm{E}-05$ \\
& QSNGS & 218147 & 1194.31 & $8.43 \mathrm{E}-05$ \\
\hline
\end{tabular}


Table 3. Numerical result of Example 3

\begin{tabular}{clrrc}
\hline$M$ & Method & \multicolumn{1}{c}{$\ell_{\max }$} & \multicolumn{1}{c}{$s$} & $\varepsilon_{\max }$ \\
\hline 256 & NGS & 24325 & 16.70 & $2.71 \mathrm{E}-06$ \\
& HSNGS & 7082 & 5.85 & $2.90 \mathrm{E}-06$ \\
& QSNGS & 2015 & 3.72 & $2.88 \mathrm{E}-06$ \\
\hline 512 & NGS & 81729 & 113.26 & $1.86 \mathrm{E}-06$ \\
& HSNGS & 24325 & 47.89 & $2.71 \mathrm{E}-06$ \\
& QSNGS & 7082 & 6.61 & $2.90 \mathrm{E}-06$ \\
\hline 1024 & NGS & 265698 & 767.23 & $3.33 \mathrm{E}-06$ \\
& HSNGS & 81729 & 321.39 & $1.86 \mathrm{E}-06$ \\
& QSNGS & 24325 & 51.35 & $2.71 \mathrm{E}-06$ \\
\hline 2048 & NGS & 882282 & 5164.64 & $1.66 \mathrm{E}-05$ \\
& HSNGS & 265698 & 1031.30 & $3.33 \mathrm{E}-06$ \\
& QSNGS & 81729 & 343.43 & $1.86 \mathrm{E}-06$ \\
\hline 4096 & NGS & 2853985 & 33726.52 & $6.10 \mathrm{E}-05$ \\
& HSNGS & 882282 & 5601.43 & $1.66 \mathrm{E}-05$ \\
& QSNGS & 265698 & 1772.49 & $3.33 \mathrm{E}-06$ \\
\hline
\end{tabular}




\section{Table 4. Arithmetic operations per iteration}

\begin{tabular}{lcc}
\hline \multirow{2}{*}{ Method } & \multicolumn{2}{l}{ Arithmetic operations per iteration } \\
\cline { 2 - 3 } & PLUS/MINUS & MULTIPLY/DIVIDE \\
\hline NGS & $\mathbf{2}(\boldsymbol{M}-\mathbf{1})$ & $\mathbf{3}(\boldsymbol{M}-\mathbf{1})$ \\
\hline HSNGS & $\mathbf{2}\left(\frac{\boldsymbol{M}}{\mathbf{2}}-\mathbf{1}\right)$ & $\mathbf{3}\left(\frac{\boldsymbol{M}}{\mathbf{2}}-\mathbf{1}\right)$ \\
\hline QSNGS & $\mathbf{2}\left(\frac{\boldsymbol{M}}{\mathbf{4}}-\mathbf{1}\right)$ & $\mathbf{3}\left(\frac{\boldsymbol{M}}{\mathbf{4}}-\mathbf{1}\right)$ \\
\hline
\end{tabular}

\section{Acknowledgement}

The authors gratefully acknowledge that this paper was supported by the university research grant scheme (GA19061) from Universiti Malaysia Sabah, Malaysia.

\section{References}

[1] A. Sunarto, J. Sulaiman, Investigation of fractional diffusion equation via QSGS iterations, J. Phys. Conf. Ser., 1179 (2019), Art. 012014.

[2] A.D. Polyanin and V.F. Zaitsev, Handbook of Nonlinear Partial Differential Equation, Chapman and Hall/CRC Press, Boca Raton (2004).

[3] A.M. Wazwaz, The variational iteration method: A powerful scheme for handling linear and nonlinear diffusion equations, Comput. Math. Appl., 54 (2007), 933-939.

[4] E. Aruchunan, M.S. Muthuvalu, J. Sulaiman, Quarter-Sweep iteration concept on conjugate gradient normal residual method via second order quadrature - finite difference schemes for solving Fredholm integrodifferential equations, Sains Malaysiana, 44, No 1 (2015), 139-146.

[5] J.H. Eng, A. Saudi, J. Sulaiman, Implementation of Quarter-Sweep approach in poisson image blending problem, In: R. Alfred, Y. Lim, A. 
Ibrahim, P. Anthony (Eds), Computational Science and Technology, Lecture Notes in Electrical Engineering, Vol. 481, Springer, Singapore (2019).

[6] J.J. More, Global convergence of Newton-Gauss-Seidel methods, SIAM J. Numer. Anal., 8, No 2 (1971), 325-336.

[7] J.L. Vazquez, The Porous Medium Equation: Mathematical Theory, Oxford University Press, New York (2007).

[8] J.M. Ortega, W.C. Rheinboldt, Monotone iterations for nonlinear equations with application to Gauss-Seidel methods, SIAM J. Numer. Anal., 4, No 2 (1967), 171-190.

[9] J.V.L. Chew, J. Sulaiman, Half-Sweep Newton-Gauss-Seidel for implicit finite difference solution of 1D nonlinear porous medium equations, Global J. Pure Appl. Math., 12, No 3 (2016), 2745-2752.

[10] M. Othman, A.R. Abdullah, An efficient four points modified explicit group Poisson solver, Int. J. Comput. Math., 76 (2000), 203-217.

[11] M.N. Suardi, N.Z.F.M. Radzuan, J. Sulaiman, Performance analysis of Quarter-Sweep Gauss-Seidel iteration with cubic b-spline approach to solve two-point boundary value problems, Adv. Sci. Lett., 24, No 3 (2018), 17321735.

[12] N.M. Nusi, M. Othman, Half- and Quarter-Sweeps implementation of finite-difference time-domain method, Malaysian J. Math. Sci., 3, No 1 (2009), 45-53. 